\title{
When will we escape from the medical funding industry's 'spin'?
}

To the Editor: I am a specialist in private practice. A patient recently asked for a quotation for an operation, and I supplied her with the relevant codes and pricing structures. As we have no contracts with medical aids she was advised to contact her insurer to ascertain which payments she could expect. Obtaining this information from them was a challenge. She was informed that they could not provide an estimate as to which part of the cost they would cover (thankfully, she was not regaled with the usual 'we refund 100\%, 200\% and even $300 \%$ of the bill'). She was also told that they had not heard of code 0023, which is the anaesthetic modifier for time that must feature on an anaesthetic account and with which most funders are familiar.

This anecdote illustrates the wilful or unintentional incompetence of the medical funding industry. At the same time we are bombarded with media reports from the Minister of Health, the Editor of the $S A M J$ and the Board of Healthcare Funders expressing alarm at escalating healthcare costs and the integral part the private sector plays in this.

Health practitioners in private practice should have a fee structure as prescribed by their peers. I believe that this is the norm in other professions, and do not see why doctors should be treated differently. Fees may sometimes be dictated by harsh market forces of supply and demand, but certainly not by the Minister of Health, the Health Professions Council of South Africa, or the funding industry.

Patients are expected to pay a hefty monthly premium to an outside institution that promises to cover their medical costs. They unquestioningly accept this promise, but increasingly it remains unkept. However, promises broken by funders have mutated into overcharging by providers.

Although it is well publicised that medical aids are non-profit organisations, administrative profits seem to be the reason why medical aids such as Discovery are doing very successful business.

Has the time not come to stop the tired refrain of 'doctors overcharging' and focus on an industry that adds little of value to its members (patients) and is increasingly part of the problem rather than part of the solution?

Patients approach us for quotes, which are provided. This information can be studied and terms discussed with the service provider, a process generally well accepted by patients. When medical aid comes on the scene, patients expect it to cover everything and question why medical aid rates are not charged. The obvious response to this logic is that medical aids would probably not be impressed if doctors suggested monthly premiums they should charge!

In short: doctors and patients should discuss and negotiate fees between themselves. There is no need for medical aid assistance or interference. Patients entrust doctors with matters that may involve life or death. Maybe the Minister, the HPCSA and the BHF should trust us to write a fair account?

Leon Groenveld

Honeydew, Johannesburg

legro@iafrica.com 